\title{
A REVIEW ON FAST DISSOLVING TABLET
}

\section{KALINDI CHAUHAN ${ }^{*}$, RAKESH SOLANKI ${ }^{1}$, SHIVANI SHARMA ${ }^{2}$}

\author{
1Department of Pharmaceutics, 2Department of Quality Assurance, Mandsaur University, Mandsaur (M. P.)
}

Email: kalindi2591@gmail.com

Received: 26 Jun 2018, Revised and Accepted: 04 Sep 2018

\begin{abstract}
In the present scientific scenario, the drug delivery technology has become extremely competitive and quickly evolving with ever-increasing demand. Fast dissolving tablet (FDT) is one such style of an innovative and distinctive drug delivery system. Once FDT is placed on the tongue, it disintegrates or dissolves quickly (in seconds) without chewing or water. Fast dissolving pills became well-liked because of higher patient compliance and most popular over typical capsules and tablets. Numerous FDT products entered the market in the nineteen eighties. This novel drug delivery like FDT or mouth dissolving tablet (MDT) has overcome several disadvantages like dysphagia or non-accessibility of water whereas travel. Compared with typical dosage form FDT is an alternative as well as helpful for the patient. The basic approach employed in the development of FDT is that the use of superdisintegrants like crosspovidone, croscarmellose sodium or maximizing pore structure within the formulation. This review article contains different techniques used for preparing FDT, silent features, numerous proprietary technologies, mechanism of super disintegration, and also the limitations.
\end{abstract}

Keywords: Fast dissolving tablet, Mouth dissolving tablet, Dysphasia, Superdisintegrants, Crosspovidone, Croscarmellose sodium

(C) 2018 The Authors. Published by Innovare Academic Sciences Pvt Ltd. This is an open access article under the CC BY license (http://creativecommons.org/licenses/by/4.0/] DOI: http://dx.doi.org/10.22159/ijap.2018v10i6.28134

\section{INTRODUCTION}

Solid dosage forms are common due to easy administration, correct dosage, self-medication, pain rejection and most significantly the patient compliance. The foremost common solid dosage forms are being tablets and capsules; one vital disadvantage of this dosage forms for a few patients is that the problem to swallow. Drinkable plays a vital role in the swallowing of oral indefinite quantity forms. Usually individual's expertise inconvenience in swallowing typical dosage forms like the pill, once water is not offered, within the case of nausea, and unexpected episodes of coughing throughout the respiratory disorder, allergic condition and respiratory illness. For these reasons, tablets which will quickly dissolve or disintegrate within the mouth have attracted an excellent deal of attention [1].

The issue of gulping could be a typical advancement in an exceedingly geriatric patient because of the stress of stifling, hand tremors, dysphasia and in youngsters because of immature strong and sensory systems and in schizophrenic patients that winds up in poor patient consistency. Close to third of the population (mainly pediatrics and geriatric) has swallowing difficulties, leading to poor compliance with an oral pill drug medical care that ends up in reduced overall medical care effectiveness [2].

There has been an increased demand for a lot of patients-convenient and compliant dose forms in the marketplace for the past few decades, due to that the demand for developing new technologies has been increasing day by day. Because the development value of a replacement drug molecule is extremely high, efforts are created by pharmaceutical industries and researchers to concentrate on the event of latest drug dosage forms for existing one having improved safety and effectiveness with reduced dosing frequency and a lot of value effective dose forms. For many therapeutic agents accustomed manufacture general effects, the oral route represents the foremost most well-liked method of administration due to its many advantages and high patient compliance compared to different routes [2].

United States Food and Drug Administration (USFDA) outlined FDT as "A solid dose type containing a medicative substance or active ingredient that disintegrate quickly sometimes in a matter of seconds once placed upon the tongue" [3].

The various synonyms used for FDTs embody mouth dissolving tablets (MDTs), orally disintegrating tablets (ODTs), melt-in-mouth tablets, and quick dispersion pill, Orodispersible, fast dissolving and fast disintegrating tablets [1].

\section{Criteria for quick dissolving drug delivery system}

$>$ The tablet must not need water to swallow however, it ought to dissolve within the mouth in a matter of seconds.

$>$ Be perfect with taste covering.

$>$ Be transportable while not fragility concern.

$>$ Have an agreeable mouth feel.

$>$ Display low susceptibility to a condition as temperature and moistness.

$>$ Leave least or no remnant inside the mouth once oral administration.

$>$ Allow the appropriate pill producing process and packaging equipment's at low value [1].

Salient features of fast dissolving drug delivery system

$>$ Ease of administration for patients who are unstable, disabled and uncooperative.

$>$ No demand of water to the patient.

$>$ It ought to be the quick disintegration of fast dissolving in nature.

$>$ Overwhelm unsatisfactory taste of the drug.

$>$ Can be designed to leave lowest or no residue within the mouth once administration and additionally to produce a nice mouth feel.

$>$ It permits high medication stacking.

$>$ Ability to create advantages of the fluid solution inside the sort of strong preparation [4-5].

\section{Advantages}

$>$ Patient having problem in swallowing like medicine, geriatric and psychiatrically patients will simply administer this kind of dosage form.

$>$ Improved quiet consistence.

$>$ Rapid begins of activity and ought to give an enhanced bioavailability.

$>$ Comfort of taking and precise dosing when contrasted with fluids. 
> Gives correct dosing as compared to liquids.

$>$ Free need to measure, an important disadvantage in liquids.

$>$ Better stability.

$>$ No need of water to swallow the measurement from, that is to a great degree helpful component for patients who are voyaging and don't have prompt access to water.

$>$ Good mouth feel property fluctuates the basic perspective of the drug as "bitter drug".

$>$ Some medicines are absorbed from the mouth, throat and oesophagus because the secretion passes down into the abdomen, and in such cases bioavailability of the medication in exaggerated.

$>$ Pregastric assimilation may bring about enhanced bioavailability enhanced clinical execution through a markdown of undesirable impacts.

$>$ The oral or buccal membrane being extremely vascularized, medicines are often absorbed directly and may enter the circulation while not undergoing fpipsts internal organ metabolism [6-8].

\section{Disadvantages}

$>$ The disadvantage is that they are fragile and brittle.

$>$ It wants a special package for defense throughout storage and transportation.

\section{Limitations}

$>$ The tablets once in a while have lower mechanical quality. Hence, careful handling is needed throughout producing method.

$>$ The tablets may leave a disagreeable taste as well as abrasiveness in mouth if not grew appropriately.

$>$ Drugs with bigger measurements are hard to formulate into FDT e. g. Rifampin (600 mg), ethambutol $(1000 \mathrm{mg})$ etc.[6-8]

\section{Ideal properties of FDTs}

They should-

$>$ Not need water to swallow and may dissolve or disintegrate within the mouth inside many seconds.

$>$ Permit high medication stacking.

$>$ Be good with taste covering and diverse excipients.

$>$ Have a delightful mouth feel.

$>$ Leave little or no deposit inside the mouth.

$>$ Have adequate strength to resist the trials of the producing method and

$>$ Post producing, handling.

$>$ Exhibit low affectability to natural conditions like stickiness and temperature.

$>$ Be adaptable and agreeable to existing procedure.

$>$ Permit the fabricate of tablets utilizing standard process and equipment at low Cost [9-10].

\section{Selection of excipients}

The property of the active ingredients in fast-dissolving tablets is balanced by excipients. This requests a concentrated comprehension of the science of those excipients to stop connection with the actives. These inactive food-grade ingredients, once incorporated within the formulation, impart the specified organoleptic properties and products effectivity.

\section{$>$ Bulk agents}

Quick dissolving tablets arrangement bulking agent are imperative. This upgrades the physical characteristics, improves the crumbling inside the mouth and decreases the convergence of the dynamic compound within the composition. The building specialists for this conveyance framework should be extra sugar-based like polydextrose, mannitol, lactitol, DCL and starch hydrolystate for higher liquid solubility and good sensory perception. Mannitol most importantly has high fluid dissolvability and great sensory perception. Bulking agents are additional within the range of ten percent to concerning ninety percent by weight of the ultimate composition. The excipients may be ranked in descendant order in terms of their brittleness: crystalline polysaccharide>spray-dried lactose $>$ beta lactose $>$ alpha lactose $>$ alpha lactose monohydrate $>$ di calcium phosphate dihydrate.

The sugar essentially based excipients that are normally utilized as especially building operators (like dextrose, fructose, maltose, mannitol, sorbitol, starch hydrolysate, polydextrose and xylitol) that show high fluid dissolvability and sweetness, and in this manner bestow style veiling property.

Sugar-situated excipients on the basis of molding and dissolution fee.

Form one saccharides (lactose and mannitol) showcase low mold capacity nevertheless excessive dissolution rate. Type a pair of saccharides (maltose and malitol) show off high mold capability however the low dissolution rate [14-15].

\section{$>$ Emulsifying agents}

A large range of emulsifiers is suggested for fastdissolving tablet formulation, Emusifying agent are obligatory excipients for formulating fast dissolving tablets they avail in expeditious disintegration and drug release without masticating, swallowing or drink. Additionally, emulsifying agent's addition is good at stabilizing the immiscible mixes and improving bioavailability. Examples-alkyl group sulfates, propylene glycol esters, lecithin, sucrose esters and others. These agents are regularly consolidated within the vary of $0.05 \%$ to about $15 \%$ by weight of the final composition.

\section{$>$ Lubricants}

It will formulate the tablets more palatable after they disintegrate within the mouth. Lubricants take away grittiness and advantage within the drug conveys mechanism from the mouth down into the stomach. Some lubricating agents are stearic acid, Mg stearate, Zinc stearate, talc, polyethylene glycol, liquid paraffin, magnesium lauryl sulfate, colloidal silicon oxide [14-15].

\section{$>$ Flavors and sweeteners}

Flavors and taste-masking agents build the product addition palatable and pleasant feel for patients. The additament of those ingredients adavantage in surmounting acerbity and discrepant tastes of some active ingredients. Each natural and synthetic flavors are often used to upgrade the organoleptic characteristics of FDTs. Peppermint flavor, flavoring aromatic oil, peppermint oil, clove oil, bay oil, anise oil, eucalyptus oil thyme oil, oil of acerbic almonds. Flavoring agents include vanilla, citrus oils, fruit essences. Formulators can make a pick from a good range of sweeteners together with sugar, dextrose,and fructose, as well as non-nutritive sweeteners such as aspartame, sodium saccharin, sugar alcohols and sucralose.

\section{$>$ Superdisintegrants}

Tablet formulations and in capsule formulations disintegrants are substances commonly included to promote moisture perforation and dispersion of the matrix of a dosage form in dissolution fluids. Super disintegrants are typically utilized at a low concentration, customarily $1-10 \%$ by weight relative to total weight of dosage unit.

Ideally, these agents should cause the tablet to disintegrate, not only into the granules from that it absolutely was compressed however supplementally into powder particles from that the granules were formulated. Typically used superdisintegrants are cross-linked polymer and cross linked starch respectively microcrystalline cellulose, croscarmellose sodium (Ac-Di-Sol), Crosspovidone (CP), sodium starch glycolate (SSG), modified corn starch, pregelatinzed starch, sodium starch glycollate, sodium carboxy-methyl cellulose, 
calcium carboxy-methyl cellulose. Sodium starch glycollate has great flowability than crosscarmellose sodium. Cross povidone is stringy nature and very compactable.

\section{- Mechanism of superdisintegrants}

Solid dosage forms adequacy enhance by utilizing Superdisintegrants. This can be achieved through numerous mechanisms. The mechanism by that the tablets are broken into little items and so produces a homogenized suspension is predicated on:

\section{Swelling}

Although water penetration could be a necessary initiative for disintegration, swelling is maybe the foremost wide accepted mechanism of action for pill disintegrants. Particles of disintegrants swell on coming back in-tuned with appropriate medium and a swelling force develops that result in the break-up of the matrix. Tablets with high consistency indicate poor breaking down due to an absence of sufficient swelling power. On the contrary hand, swelling power is applied inside the pill with low consistency. It's worthy to note that if the pressing part is to a great degree high, liquid can't infiltrate inside the pill and breaking down is again backs off.

\section{Porosity and surface tension (wicking)}

Effective disintegrants that don't swell are showing their disintegrating action through porosity and surface tension. Pill porosity gives pathways to the penetration of liquid into tablets. When we put the pill into appropriate liquid medium, the medium enter into the pill and replaces the air absorbable on the particles that debilitates the intermolecular bond and breaks the pill into fine particles. Hydrophilicity of the medication/excipient chooses the water take-up by tablet. A hydrophilic network around the drug particles helps in disintegration. Repulsive powers of particles system of crumbling clarify that the 'non-swellable' disintegrants made swelling of pill. A molecule aversion hypothesis upheld that non-swelling molecule additionally causes breaking down of tablets. The principle component is electrical repulsive powers between particles.

\section{Deformation}

Disintegrated particles get deformed throughout tablets compression and once these distorted particles are available to contact with liquid media or water, they get into their traditional structure. Pressure enhances the swelling ability of starch owing to this increase in size of the distorted particles produce a breakup of the pill [11-15].

\section{- Surface active agents}

Sodium doecyl sulfate, sodium lauryl sulphate, polyoxy ethylene sorbitan fatty acid esters (Tweens), sorbitan fatty acid esters (Spans), polyoxy ethylene stearates.

\section{- Binder}

Polyvinyl pyrrolidone (PVP), polyvinyl alcohol (PVA), hydroxy propyl methyl cellulose (HPMC).

\section{- Colour}

Sunset yellow, Amaranth etc.

\section{Mechanism}

Bioavailability of a medication relies upon ingestion of the medication that is solubility of the medication in git liquid and penetrability of the medication crosswise over git layer. The dissolvability of a medication essentially relies upon physiochemical properties of the medication. The speed of medication disintegration is incredibly impacted by deterioration of the pill. Disintegrants are necessary excipient of the pill formulation, \{they are invariably additional to pill to induce breakup of pill after they are comes involved with liquid fluid and this method of group action of constituent particles before the drug dissolution happens, is thought as disintegration method and excipients that induce this method are referred to as disintegrants. Disintegrants are to expand the zone of the pill sections and to control a cohesive force of particles [16].

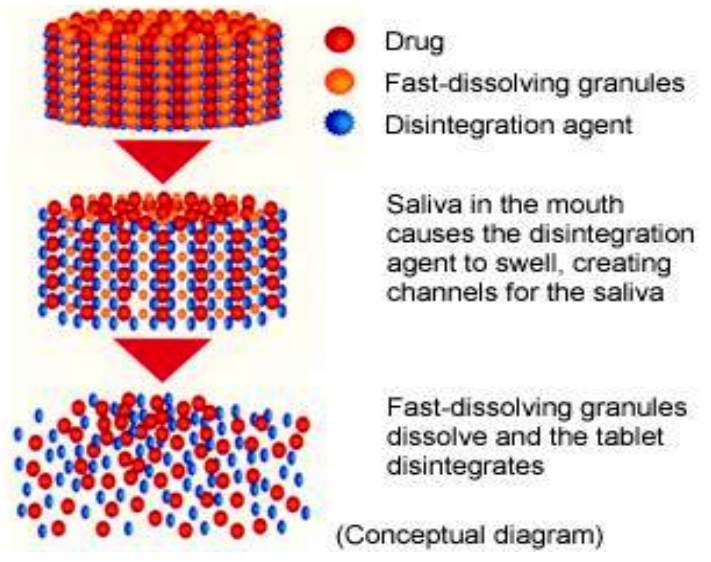

Fig. 1: Mechanism of fast dissolving tablet [17]

Technologies used to manufacture fast dissolving tablets conventional technologies

\section{Freeze-drying or lyophilization}

It is the strategy inside which water is sublimated from the item when it's solidified by freezing. This system creates an amorphous porous structure that may dissolve quickly [17-19].

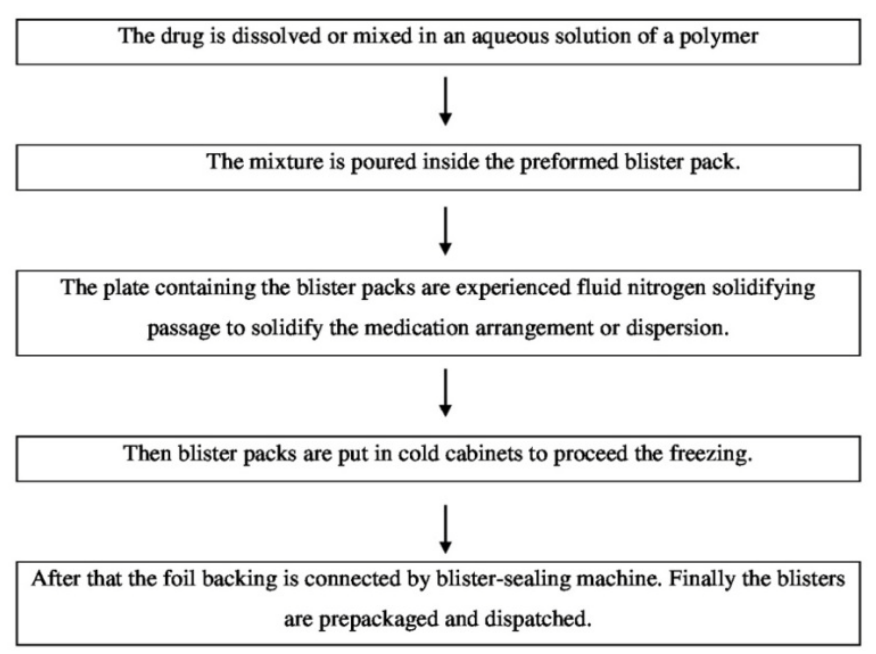

Fig. 2: Systemic representation of freeze drying process [36] 


\section{Advantages}

- Improved absorption and increase in bioavailability.

- Tablets made by this method possess terribly low disintegration time. Render tablets with nice mouthfeel as a result of quick melting result.

- Gives quick disintegration (5 sec).

- Lyophilization is advantageous for warm a delicate medication that is thermolabile substances.

- Expands absorption and bioavailability of medication.

- Tablets ready by freeze-drying disintegration quickly in but five sec as a result of fast penetration of spittle in pores once placed in mouth.

\section{Disadvantages}

- Relatively costly and time overwhelming method.

- The item acquired is inadequately stable and delicate, sensitive to wetness rendering common packaging unsuitable.

- Very poor physical resistance underneath stressed conditions.

- High value of production [20].

\section{$>$ Tablet molding}

Molding process is of two types-solvent method and heat method.

\section{- Solvent method}

Solvent method involves

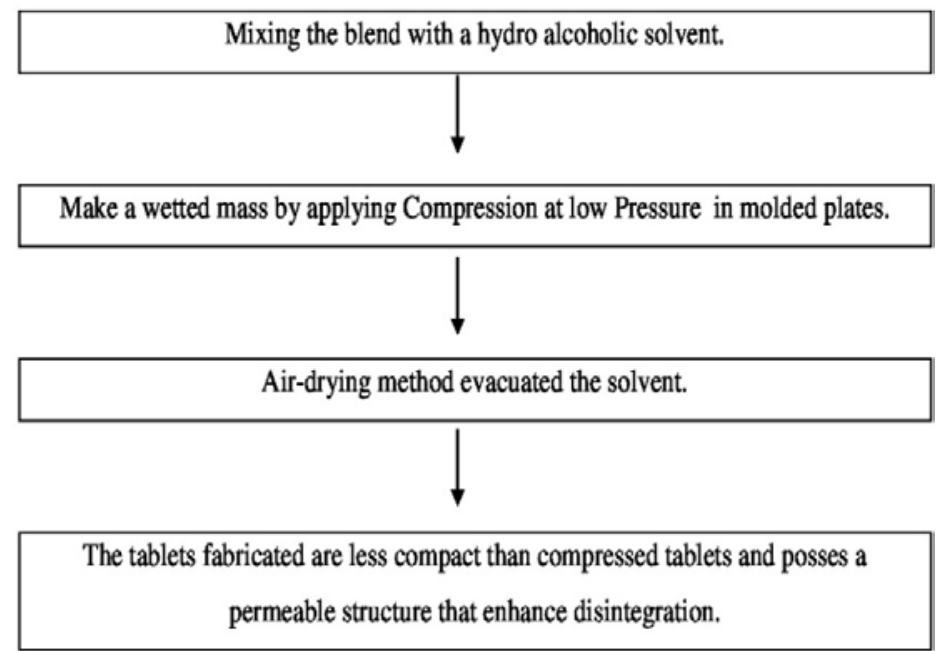

Fig. 3: Systemic representation of solvent method [36]

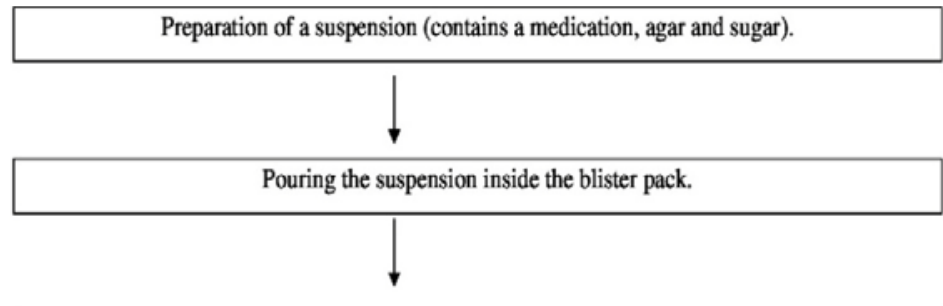

Solidifying the agar at the room temperature to create a jelly \& drying at $300 \mathrm{C}$ beneath vacuum

Fig. 4: Systemic representation of heat method [36]

\section{- Heat method}

The heat molding process involves

Masking of taste is a new drawback to the present technology and therefore the masked drug particles are ready by spray congealing a melted mixture of modify polyethylene glycol, vegetable oil, lecithin, and sodium carbonate an active ingredient into a lactose-based Tablet triturate type. Tablets made by the moulding technique is simple to rescale for the industrial manufacturer, compared to the lyophillisation technique [21-23].

\section{$>$ Spray drying}

In this technique, formulation contained gelatin can be used as a matrix and as a supporting agent, mannitol as a bulking agent and lactose, crosscarmellose, cross povidone, sodium starch glycolate are used as superdisintegrants. Tablets made from the spray dried powder have been demonstrated quick dissolution and upgraded disintegration. It breaks down in less than 20 seconds in watery medium [24]

\section{$>$ Direct compression}

Direct compression represents the best and most value effective tablet producing technique. During this technique, tablets are ready directly by compression of the mixture of drug and excipients with none preliminary treatment [26].

\section{Advantages}

- High doses will be accommodated and the final weight of the tablet will exceed that of other strategies.

- Most effortless approach to fabricating the tablets.

- Conventional instrumentation and normally accessible excipients are used.

Cost-effectiveness [27]. 


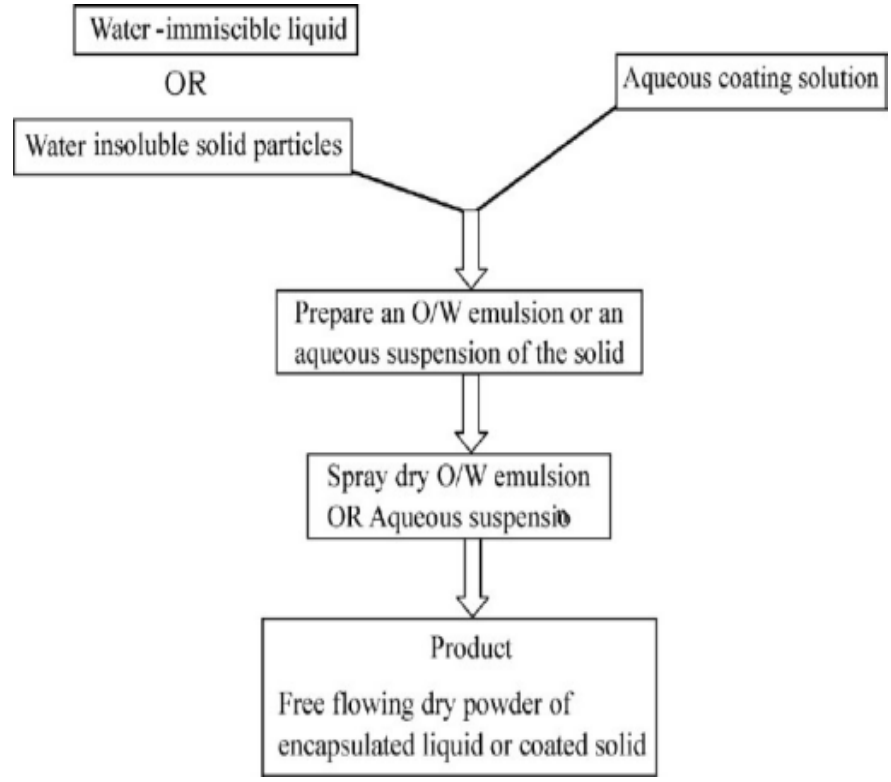

Fig. 5: Spray drying technique [25]

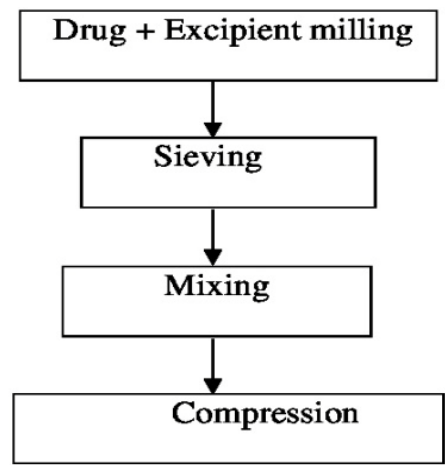

Fig. 6: Systemic representation of direct compression [26]

\section{Sublimation}

To generate a porous matrix, volatile ingredients are incorporated within the formulation that is later subjected to a method of sublimation extremely volatile ingredients like ammonium bicarbonate, ammonium carbonate, benzoic acid, camphor, hydrocarbon, urea, ester and anhydride could also be compressed alongside alternative excipients into a pill. This volatile material is then evacuated by sublimation leaving a to a great degree polymer matrix [28].

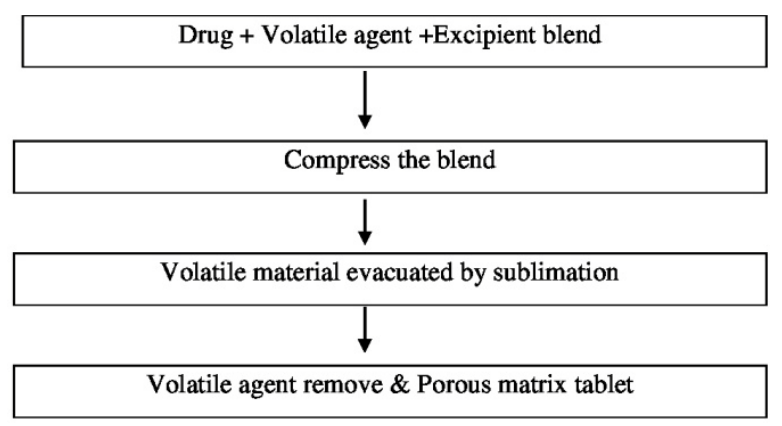

Fig. 7: Systemic representation of sublimation [6]

\section{$>$ Cotton candy process}

This procedure is so named as it uses a special turning system to create floss like crystalline structure, which imitate cotton Candy. Cotton candy process includes the arrangement of Matrix of polysaccharides or saccharides by the synchronous activity of flash melting and spinning. The network shaped is mostly recrystallized to have enhanced flow properties and compressibility. This candy floss network is then blended with drugs and excipients and along these lines packed to fast dissolving tablet [29]. 


\section{$>$ Mass extrusion}

This technology involves softening the blend using the solvent mixture of water-soluble substance like polyethylene glycol, methyl alcohol and removal of diminished mass through the extruder or syringe to instigate a barrel of the item into even sections utilizing warmed sharp edge to make tablets. The drying chamber may likewise be utilized to coat granules of unpleasant tasting drug and thereby masking their bitter taste [30].

\section{$>$ Patented technologies}

\section{- Zydis technology}

Zydis ${ }^{\circledR}$ was presented By R. P. Scherer Corporation in 1986. This method drug to be dissolved or dispersed in an aqueous solution to form soluble blend then the blend is filled into the preformed blister pockets and freeze-dried. This prompts a tablet that crumbles in the mouth in seconds. The 2 most regularly utilized basic added substances are gelatin and mannitol however, another (example starches, gums, etc.) Could likewise be utilized relying upon the properties of the drug. As a general rule, the most effective physical characteristics are achieved by using a mixture of a soluble compound and a crystalline sugar alcohol or amino acid at a typical combined concentration of 100 percent $w / w$ within the matrix solution. The polymer gives the quality and flexibility through the crystalline part gives the hardness and surface [31]. Zydis system is a freeze-dried tablet where drug is entrapped or dissolved within the matrix includes fast-dissolving material. Once zydis units are placed into the mouth, unit disintegrates instantly and doesn't need water to help to swallow. The zydis matrix consists of the many materials designed to attain a variety of objectives. To give quality to get crystallinity, elegance, and hardness like alginates, gelatin, dextran are consolidated, saccharides like sorbitol or mannitol are joined. Water is employed within the manufacturing method to make the sure production of porous units to attain fast disintegration. Various gums are utilized to prevent sedimentation of dispersed medication particles inside the manufacturing method. Crumple protectants like glycine stop the shrinkage of zydis units all through a lyophilization technique or long storage. Zydis item is pressed in blister packs to defend the plan from dampness inside the environment.

\section{Limitations}

- The quantity of drug may be incorporated ought to usually be but $400 \mathrm{mg}$ for insoluble drug and less than $60 \mathrm{mg}$ for soluble drugs.

- The particle size of the insoluble drugs should not be less than $50 \mu \mathrm{m}$ and not more than $200 \mu \mathrm{m}$ to stop deposit throughout the process [32].

\section{Advantages}

- The buccal tubular cavity and internal organ regions are all areas of absorption from this formulation. Any pre-gastric absorption avoids first-pass metabolism and may be a plus in medication that ensures an excellent deal of hepatic metabolism.

- The zydis formulation self-preserving as a result of the ultimate water concentration within the freeze-dried product is simply too low to permit for microbic growth.

\section{Disadvantages}

- The method of lyophilization may be a comparatively costly manufacturing method.

- The formulation is extremely light-weight and fragile, and so should not be kept in backpacks or the lowest of handbags.

- It has poor stability at more temperatures and humidities.

- The lyophilization is a time severe system. It has poor physical resistance.

- $\quad$ Loading of a high dose of soluble medication is not potential [33].

\section{- Durasolv technology}

DuraSolv is Cima's second-generation fast-dissolving/disintegrating pill formulation like OraSolv, DuraSolv has a lot of higher mechanical strength than its precursor because of the utilization of upper compaction pressures throughout tableting.

\section{Advantages}

- Durasolv tablets are formulated by utilizing conventional tableting and show great $2 \%$ friability.

The durasolv product is cost-effective as good as a faster manufacturing product.

- Durasolv is long lasting seeing that it is prepackaged in blister packaging, pouches or vials.

- The durasolv innovation is most appropriate to plans and similarly little dosages of the drug.

\section{Disadvantages}

- One disadvantage of this science will not be compatible with higher doses of a drug, for that reason of the formula is subjected to such excessive pressures on compaction. Unlike orasolv, the structural integrity of any style masking can also be compromised with excessive drug doses.

- The drug powder coating in durasolv would grow to be damaged due to compaction, unlock the bitter-tasting drug to a sufferer's style buds [34].

\section{- Orasolv technology}

CIMA labs have created Orasolv Technology. In the course of this method, drug taste masked. It furthermore carries the effervescent disintegrating agent. Tablets are created by means of direct compression process at low compression drive so that you could decrease oral dissolution time. Standard blenders and tablet machine are employed to produce the tablets. The tablets made are delicate and friable [3, 35]. Effervescent agent is that the main ingredient utilized in this technology. The drug microparticles are gently compressed together with the effervescent agent. The developed pills have the look of a standard compressed tablet However, they are weaker and additional fragile than the standard tablets. Thus, there is a demand for a special packaging. The particle coating that is employed for taste masking purpose is not cracking at the time of compression due to a low compression force [22-36].

\section{- Wow technology}

It is proprietary by Yamanouchi Pharmaceutical Corporation wherever wow tends for "without water". During this method high mould ability carbohydrate like saccharide, a diuretic is mixed with low mouldability carbohydrate like glucose, disaccharide, and mannitol to get quickly melting tablet [37-39].

\section{- Flashdose technology}

This technology is much like candy floss, employing a distinctive spinning mechanism to provide crystalline floss structure. The medication will then be fused into this crystalline sugar and packed into a pill. Such product contains a high area for dissolution, dissolving quickly on tongue and simple dispersion. The Flash dose pills involves self-binding shear kind matrix termed as "floss" [36].

\section{- Flashtab technology}

This technology aims to form the drug have fast release in git, small encapsulated drug with effervescence, and simply flash diffusion pill. Typically the chemical compound used is Eudragit for fast release. This technology uses standard approach of wet/dry granulation follow by classical methodology of compression. Micro granules of medication, taste masking agents, disintegrating agent, and swelling agents are utilized to formulate medicine. These tablets have smart physical resistance, and extremely recommended for hygroscopic materials for blister packing as materials like PVC/aluminum foils cater higher moisture protection compared to traditional polyvinyl chloride or polypropylene foils [38-39].

\section{CONCLUSION}

Due to the increasing demand for novel drug delivery, the quickdisintegrating drug delivery a system has become one in the entire 
milestone within the novel drug delivery system. The introduction of quick-dissolving drug delivery system has encountered the delivery of standard dosage form.

\section{AUTHORS CONTRIBUTIONS}

The entire authors have contributed equally

\section{CONFLICT OF INTERESTS}

Declared none

\section{REFERENCES}

1. Bhowmik D, Chiranjib B, Krishnakanth P, Chandira RM. Fast dissolving tablet: an overview. J Chem Pharm Res 2009;1:163-77.

2. Sharma R, Rajput M, Pawan P, Sharma S. Review article fast dissolving drug delivery system. Int Res J Pharm 2011;2:21-9.

3. Siddiqui MN, Garg G, Sharma PK. Fast dissolving tablets: preparation, characterization and evaluation: an overview. Int J Pharm Sci 2010;4:87-96.

4. Deshmukh VN. A Mouth dissolving drug delivery system. Int J Pharmtech Res 2012;2:412-21.

5. Reddy LH, Ghosh B. Fast dissolving drug delivery systems: a review of the literature. Int J Pharm Sci 2002;64:331

6. Harish VD, Valli G, Ramya MG. A review on fast dissolving tablets. Int J Univers Pharm Bio Sci 2014;3:757-81.

7. Sastry SV, Nyshadham JR, Fix JA. Recent technological advances in oral drug delivery-a review. Pharm Sci Technol Today 2000;3:138-45.

8. Patil SL, Shivnikar MA. Formulation and technology of fast disintegrating tablets: a review. J Pharm Biomed Sci 2011;9:1-7.

9. Nand P, Vashist N, Anand A, Drabu S. Mouth dissolving tablets-a novel drug delivery system. Int J Appl Biol Pharm Technol 2010;1:XX.

10. Shukla D, Chakraborty S, Singh S, Mishra B. Mouth dissolving tablets I: an overview of formulation technology. Sci Pharm 2009;77:309-26.

11. Pahwa R, Gupta N. Superdisintegrants in the development of orally disintegrating tablets: a review. Int J Pharm Sci Res 2011;2:2767.

12. Alam NM, Sharma S, Jaimini M, Mohan Shelendra, Chatterjee A. Fast dissolving dosages form: boon to emergency conditions. Int J Ther Appl 2014;16:1-7.

13. PS Mohanachandran, PG Sindhumol TK. Review article superdisintegrants: an overview. Int J Pharm Sci Rev Res 2011;6:105-9.

14. Panigrahi R, Behera SP, Panda CS. A review on fast dissolving tablets. Webmedcentral Qual Patient Saf 2010;1:1-15.

15. Chowdary YA, Soumya M, Madhu BM, Aparna K, Himabindu P. A review on fast dissolving drug delivery systems-a pioneering drug delivery technology. Bull Environ Pharmacol Life Sci 2012;1:8-20.

16. Ragade SM, Jain AA, Barhate S. A review on: -mouth dissolving drug delivery system. World J Pharm Pharm Sci 2017;6:467-80.

17. Yadav G, Kapoor A, Bhargava S. Fast dissolving tablets recent advantages: a review. Int J Pharm Sci Res 2012; 3:728-36.

18. Asija R, Asija S, Gupta A, Hemlata. A review on fast dissolving drug delivery system. Int J Res Pharm Sci 2014;4:7-12.
19. Indurwade $\mathrm{NH}$, Rajyaguru $\mathrm{TH}$, Nakhat PD. Novel approach: fast dissolving tablets. Indian Drugs 2002;39:405-9.

20. Nagar P, Singh K, Chauhan I, Verma M, Yasir M, Khan A, et al. Orally disintegrating tablets. J Appl Pharm Sci 2011; 1:35-45.

21. Dobetti L. Fast-melting tablets: developments and technologies. Pharm Technol Drug Delivery 2001;12:44-50.

22. Badgujar BP, Mundada AS. The technologies used for developing orally disintegrating tablets: a review. Acta Pharm 2011;61:117-39.

23. Roshan Rai R, Chirra P, Thanda V. Fast dissolving tablets: a novel approach to drug delivery-a review. Int J Preclin Pharm Res 2012;3:23-32.

24. Mizumoto T, Masuda Y, Yamamoto T, Yonemochi E, Terada K. Formulation design of a novel fast disintegrating tablet. Int $\mathrm{J}$ Pharm 2005;306:83-90.

25. Parkash V, Maan S, Deepika SK, Hemlata VJ. Fast disintegrating tablets: opportunity in drug delivery system. J Adv Pharm Tech Res 2011;2:223.

26. Jeong SH, Takaishi Y, Fu Y, Park K. Material properties for making fast dissolving tablets by a compression method. J Mater Chem 2008;18:3527-35.

27. Kaur T, Gill B, Kumar S, Gupta GD. Mouth dissolving tablets: a novel approach to drug delivery. Int J Curr Pharm Res 2011;3:1-7.

28. Mishra DN, Bindal M, Singh SK, Kumar SG. Spray dried excipient base: a novel technique for the formulation of orally disintegrating tablets. Chem Pharm Bull 2006;54:99-102.

29. Nautiyal U, Singh S, Singh R, Gopal KS. Fast dissolving tablets as a novel boon: a review. J Pharm Chem Biol Sci 2014;2:5-26.

30. Bhaskaran S, Narmada GV. Orally disintegrating tablets. Indian Pharm 2002;1:9-12.

31. Sharma SK, Sharma R, Sagar VG. Fast dissolving tablet-a review and recent advances in manufacturing technologies. Res J Pharm Dosage Forms Technol 2010;2:120-4.

32. Singh A, Masih A, Kumar A, Tiwari A. Fast dissolving tablets: a review. Int J Curr Pharm Res 2017;9:8-18.

33. Seager H. Drug-delivery products and the Zydis fast-dissolving dosage form. J Pharmpharmacol 1998;50:375-82.

34. Parashar B, Yadav V, Maurya B, Sharma L. A review article on fast dissolving tablet. Int J Appl Pharm 2012;4:17-22.

35. Kumari S, Visht S, Sharma PK, Yadav RK. Fast dissolving drug delivery system: review article. J Pharm Res 2010;3:1444-9.

36. Fu Y, Yang S, Jeong SH, Kimura S, Park K. Orally fast disintegrating tablets: developments, technologies, tastemasking and clinical studies. Crit Rev Ther Drug Carrier Syst 2004;21:433-75.

37. Puttalingaiah L, Kavitha K, Mani T. Fast disintegrating tablets: an overview of formulation, technology and evaluation. Res J Pharm Biol Chem Sci 2011;2:589-601.

38. Khanna K, Xavier G, Joshi SK, Patel A, Khanna S, Goel B. Fast dissolving tablets-a novel approach. Int J Pharm Res Allied Sci 2016;5:311-22.

39. Kumar S, Gupta SK, Sharma PK. A review on recent trends in oral drug delivery-fast dissolving formulation technology. Adv Biol Res 2012;6:6-13. 